\title{
POZOS Y MAFIOSOS. LA INACABADA TRANSICIÓN DEL MERCADO DEL AGUA A LA GESTIÓN COLECTIVA EN EL PALERMO DEL SIGLO XX
}

\begin{abstract}
RESUMEN
La creación de los consorcios de riego, en la primera posguerra, revolucionó los equilibrios sociales e hidráulicos de las regiones citrícolas de Palermo (Italia). En este artículo, a través de fuentes primarias, se analizan las relaciones conflictivas entre los agricultores usuarios, los nuevos y controvertidos organismos de gestión colectiva, los propietarios de pozos, acusados de dominar el mercado de las aguas, y los distribuidores, enjuiciados por pertenecer a la mafia en los años fascistas.

PAlabras clave: aguas subterráneas; regadío; Sicilia; consorcios de riego; mafia.
\end{abstract}

\section{Abstract}

\section{WELLS AND MAFIA. THE UNCERTAIN TRANSITION OF THE WATER MARKET TO COLLECTIVE MANAGEMENT IN THE PALERMO OF THE 20TH CENTURY}

The creation of the Irrigation Consortia, during the first post-war period, changed the social and hydraulic balances of the citricultural region of Palermo (Italy). This paper, based on primary sources, analyses the conflictive relations between the agricultural users, the new and contested institutions for collective action, the owners of the wells, accused of dominating the water market, and the water distributors, judged during the Fascist period for belonging to the mafia.

KEYwORDs: groundwater; irrigation; Sicily; irrigation consortia; mafia.

\section{InTRODUCCIÓN: AgUAS PÚbliCAS, GESTIÓN PRIVADA}

El control de los recursos hídricos, esenciales para la producción agrícola, resulta económica y socialmente central en los territorios áridos o semiáridos. La excesiva demanda, la creación de un monopolio, la ausencia de un capital social bien enraizado o los intentos de

a Geógrafo. damaro.fra@gmail.com

Fecha de recepción: septiembre 2018. Fecha de aceptación: diciembre 2018. 
regulación desde arriba pueden ser factores de conflictos violentos, pero también de impulso a la cooperación. Sin profundizar en el debate teórico sobre capital social y bienes comunes, pero teniendo en cuenta las aportaciones y de la economía institucional y de la acción colectiva (Olson, 1965; Ostrom, 1990; Agrawal, 2001), en este artículo nos centraremos en la gestión privada de las aguas subterráneas y en la creación de un organismo de gestión colectiva del riego en la provincia de Palermo, en Sicilia. Este territorio (Figura 1), la célebre Conca d'Oro, se caracterizaba por el cultivo intensivo de cítricos destinados al mercado internacional, pero también por la presencia de un tejido social mafioso que engendró el control de la distribución de las aguas subterráneas.

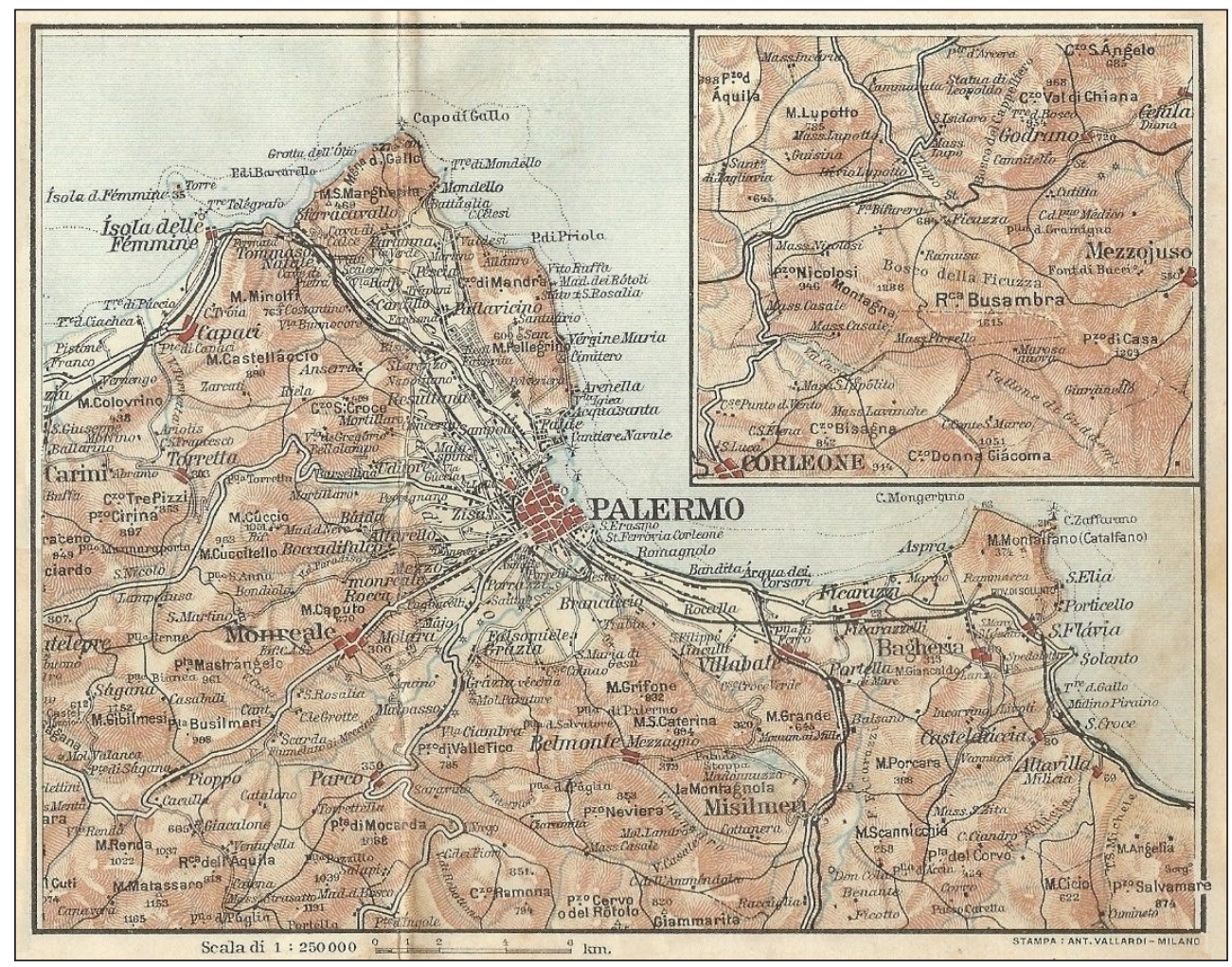

Figura 1. Área de estudio según un mapa de la época publicado por la imprenta Vallardi de Milán (1919), que muestra en amarillo claro la llanura costera dedicada al cultivo del limón entre los cabos de Gallo y Zaffarano. El río Eleuterio o Ficarazzi se encuentra en el extremo oriental del golfo de Palermo, junto a Bagheria. 
Por lo general, se habla de tres modelos de gestión del agua. En primer lugar, la administración centralizada y estatal, asociada a menudo a los estados autoritarios, como en el caso del despotismo hidráulico teorizado por Wittfogel (1966). En segundo lugar, la gestión comunitaria, es decir, de los organismos colectivos y autónomos, entre las que han destacado las organizaciones de regantes de Valencia y Murcia (Glick, 1970; Maass y Anderson, 1978; Hermosilla Pla, 2010). Sin embargo, en la gestión de las aguas subterráneas existen casos específicos muy diversos que se colocan entre esos dos extremos y ejemplos de gestión compartida entre los usuarios y las administraciones estatales — como en California—, como ponen de manifiesto Molle y Closas (2017). En tercer lugar, la gestión que Thierry Ruf (2010: 154 y 169) define como «industrial», en la que, pese a la abundancia de recursos hídricos, la privatización de los medios de abastecimiento genera un mercado del agua. Esta última — que es el modelo que más se acerca a nuestro caso de estudio— tiende a la exclusión de los campesinos más pobres.

Existe una amplia literatura agronómica sobre el regadío siciliano de pozos (Alfonso, 1877; Scrofani, 1962; Cusimano, 1990). En ella se insiste sobre el hecho que, en este territorio, la gestión del pequeño regadío era controvertida, ya que favorecía el control de los agentes privados sobre un recurso necesario. En esta zona, no existían organizaciones de propietarios para la gestión comunitaria de los recursos. Los geógrafos han destacado las repercusiones económicas negativas de este mercado del agua para los productores. El oligopolio de los proveedores les concedía un dominio social que muchos estudiosos han asociado a una gestión mafiosa de las aguas (Cusimano, 1990; Caldo, 1984). Renée Rochefort (1961) percibió, ya en los años cincuenta, que en la huerta de Palermo el agua era no sólo un elemento de progreso, sino también una herramienta para la explotación del hombre a través del miedo.

Es necesario considerar la presencia de un tejido social mafioso para entender las dinámicas productivas de la agricultura intensiva en Palermo. La mafia se configuraba como una forma de extorsión, de control penetrante de la sociedad local y de dominio ilegal de los recursos, que se adaptaba a los cambios políticos y económicos y se movía en diversos contextos institucionales. ${ }^{1}$ Los mafiosos disfrutaban de una organización compacta y centralizada capaz de cooperar con agentes externos: este capital social era, pues, una herramienta para controlar el capital físico y los recursos locales (Ostrom y Ahn, 2003: 172). El capital

1 La amplia literatura sobre la mafia siciliana ha tenido el mérito de alterar el estereotipo del problema como algo peculiar del latifundio (Verri, 2008). Ese papel de intermediación, basado en la intimidación y la violencia, se aplicó también en las zonas costeras de regadío, especialmente desde finales del siglo XIX, cuando la producción de limones se convirtió en uno de los negocios más rentables de la región. La violencia rural convivía con la huerta, con la producción de carácter internacional y con la difusión del bienestar (Pezzino, 1987; Lupo, 2004). En particular, los estudios marxistas locales de los años sesenta y setenta, situaban en las tierras de regadío de la provincia de Palermo una de las bases más importantes de la mafia, gracias precisamente al control de las aguas (Pantaleone, 1971; Drago, 1972). En este texto, no consideramos que la formación de grupos delictivos parta del sistema de riego, sino de unas dinámicas sociales previas que influyeron en la gestión de las aguas. 
social de la mafia y su capacidad de networking, según Rocco Sciarrone (1998), explicaban la creación de vínculos e intercambios, incluso con sujetos que no pertenecían a la organización. Los agricultores, buscando su protección, aceptando sus reglas y aprovechando sus servicios, reforzaban el papel de la mafia local, como apunta Salvatore Lupo (2018: 12). Esto nos ayuda a comprender la intromisión mafiosa en la producción citrícola y la presencia de un mercado de aguas que se caracterizaba por un sistema de relaciones densas y conflictivas, es decir, abusos, vejaciones y, en raras ocasiones, violencias.

Para profundizar este tema, aprovecharemos la documentación del Archivo de Estado de Palermo, producida por las autoridades fascistas entre finales de los años veinte y comienzos de los treinta, ${ }^{2}$ que evidencia esta interconexión entre poderes locales, violencia rural y regadío. En este enorme juicio por associazione a delinquere, hubo centenares de imputados: por ejemplo, en Termini Imerese y alrededores fueron 360, en Piana dei Greci 278 y, en el que tomamos como ejemplo en este texto, Bagheria y ayuntamientos lindantes fueron 361 (Lupo, 2008; Blando, 2008). Los distribuidores de agua fueron los principales acusados de pertenecer a una organización criminal y de usar el agua como herramienta de intimidación y dominio. Gracias a las investigaciones policiacas y al juicio, se confeccionó una reconstrucción detallada de las dinámicas en torno al uso de los recursos hídricos. ${ }^{3}$ Además, a través de esos documentos es posible percibir las voces — si bien tras el filtro de policía y abogadosde los actores del regadío, tanto de los que establecían los turnos, los precios y las jerarquías rurales, como de los que se declaraban víctimas de este sistema.

La administración estatal intentó responder políticamente al conflicto mediante la formación de instituciones de gestión colectiva. En este trabajo se analiza también esta acción de la administración a partir del caso del Consorzio Idro-Agricolo de Bagheria (CIAB), el más importante de la zona. Su documentación — confusa, pero abundante- es una ventana sobre los intentos de revolucionar las dinámicas productivas a través de una administración colectiva y regulada por normas consensuadas. La nueva institución se consolidó gracias a la coyuntura favorable de la citricultura, que hizo coincidir los intereses de sector con los de la región (Lupo: 1990, 262); en el marco del intervencionismo hidráulico del Estado italiano en el primer tercio del siglo XX (Stampacchia, 2000; Cavallo, 2011; Bernardi, 2014) y del discurso ruralista del régimen de Mussolini que, a partir de la segunda mitad de los años veinte, identificaba la figura del campesino con el fascismo (Lanaro, 1989: 963; Grando y Volpi, 2014). Sin embargo, en este texto, se hace hincapié en la desconfianza —no sólo inicial— de los usuarios en la administración comunitaria de las aguas. Se plantea, pues, que el éxito de

2 Archivio di Stato de Palermo, Tribunale Civile e Penale de Palermo. Procedimenti penali (giudizio). Anno 1929. N. 1086 - R. G. Trib. Associazione a delinquere. N.3240-3254. (a partir de ahora sólo «ASPa»)

3 Catedráticos y técnicos agrónomos u otros expertos declararon o presentaron informes y cartografías para describir el paisaje rural de la época y las técnicas hidráulicas. En ocasiones, exculparon a los imputados gracias a sus estudios técnicos. ASPa, b. 3245, Corte d'Appello 1930, Accertamenti, 23/07/1930, pp. 112-114. 
esa acción colectiva fue limitado por las dificultades de establecer un marco de relaciones y de consolidar un nuevo capital social basado en la reciprocidad y confianza mutua (Fonseca, Montalba y García, 2015). Las viejas costumbres de abastecimiento y la persistencia de una red social con caracteres mafiosos y oligárquicos mermaron la eficacia de los consorcios palermitanos y la creación de una economía moral de la gestión hídrica (González Alcantud, 1998; Sanchis-Ibor et al., 2017).

\section{Proveedores y USUARIOS EN LA GESTión PRIVAdA (y ¿MAFiosa?) DEL AGUA}

En el siglo XIX, en muchas regiones del sur de Italia, las aguas subterráneas eran la fuente principal para regar los cultivos intensivos de las zonas costeras. Los agricultores buscaron incesantemente una fuente de abastecimiento subterránea cercana a sus tierras, ya que las aguas superficiales eran a menudo inalcanzables (Bevilacqua, 1996: 70). Sólo se contaba con el recurso del río Eleuterio y algún otro cauce menor (Figura 1). La gestión colectiva, típica de otras regiones mediterráneas - Italia del norte inclusive- era prácticamente inexistente en Palermo. Por otra parte, con el proceso de industrialización y electrificación del campo, las bombas de extracción gestionadas por propietarios privados se multiplicaron, permitiendo la producción agrícola intensiva para el mercado internacional (Barone, 1986; Calatayud, 1990). Se trataba de un fenómeno que, tanto del punto de vista técnico, como del institucional, involucró diversos territorios del Mediterráneo, aunque con éxitos diferentes. No sólo impulsó la expansión de la citricultura, sino también la creación de nuevas instituciones de gestión, el aumento de las desigualdades (por el alto coste de las máquinas accesible sólo a los más pudientes) y la difusión de actos de violencia (Chironi et al., 1987; Ferri y Sanchis, 2001; Pardo, 2014).

En el Palermitano, la presencia de máquinas de extracción coincidió con la articulación de un mercado del agua especialmente conflictivo. Como apunta Bevilacqua (1989: 309), una bomba hidráulica, en ocasiones «extremadamente costosas», podía abastecer varias hectáreas de cítricos sicilianos. Este tipo de riego, flexible y fácil de regular, se adaptaba a las exigencias de las pequeñas empresas campesinas que producían para el voluble mercado internacional. Sin embargo, los costes sociales fueron elevados, porque el paulatino aumento de la demanda influyó en las relaciones económicas locales. Pese a la posibilidad de acceder a los recursos sin una programación a gran escala, ni necesidad de la intervención hidráulica estatal, la captación de las aguas era cara. Se trataba de un sistema descentralizado, pero sólo los propietarios más influyentes disponían de bombas propias. Incluso los que habían tenido que vender gran parte de sus tierras procuraban mantener el control de los pozos e instalar una bomba hidráulica. El agua, pues, no estaba vinculada a un territorio, es decir a la propiedad de la tierra dentro de un determinado perímetro. De esta forma, el riego se ligaba a la iniciativa individual y a una concepción privatista del recurso (Alfonso, 1875). Esta situación, 
en un contexto legal impreciso, provocaba una dialéctica conflictiva entre los proveedores de agua y los productores agrícolas (Caldo, 1973).

Faltaba un sistema de gestión centralizada o una figura administrativa estatal para el racionamiento de las aguas subterráneas. Tampoco existía una gestión colectiva privada de los pozos, que implicaría una sólida organización social y económica para invertir en las bombas, asegurar su mantenimiento y evitar las desigualdades (Molle y Closas, 2017: 121122). Ya en el último tercio del siglo XIX, la legislación del Estado italiano intentó favorecer la formación de una red hidráulica más ordenada y la formación de consorcios de riego. No obstante, como veremos, tampoco estas medidas pararon el aumento de la conflictividad que acompañó la expansión del regadío (Lupo, 1987: 90, y 1990: 46-54).

En ese contexto socio-económico y ambiental, las mismas infraestructuras, como bombas y canales, limitaron la formación de una «identidad hidráulica» caracterizada por un acuerdo sobre la distribución o una cultura colectiva de los recursos (Boelens y Vos, 2014: 59). El resultado fue que la gestión de las aguas subterráneas, basada en la propiedad privada de las bombas, generó una fuerte competencia para el acaparamiento de los turnos de riego. De hecho, los criterios de distribución no seguían una lógica de sostenibilidad hídrica o de producción agrícola: eran inconstantes y, a veces, imprevisibles. La cantidad de agua variaba según las condiciones meteorológicas, y también la demanda. Además, las necesidades hídricas del cítrico cultivado en la huerta de Palermo - los limones de primavera o verdelli que fueron un cuasi-monopolio siciliano en Europa - coincidían con el estiaje mediterráneo (Barone, 1987: 220). Por ello, la gestión privada provocaba continuas quejas entre los usuarios y entre éstos y los administradores de las máquinas. ${ }^{4}$ Estos últimos fueron algunos de los principales imputados del juicio de 1929.

Según la documentación judicial, los guardas del agua, los encargados de la gestión de las máquinas y de los canales de riego, actuaban como si fueran los «dueños» de las aguas. ${ }^{5}$ Las disputas por su control podían llevar a luchas sangrientas y, en presencia de varias fuentes, los distribuidores podían imponer los recursos más caros. Si se creaba una situación de competencia entre diversas fuentes, los guardas podían amenazar a los usuarios o destrozar los canales de los competidores. ${ }^{6}$

Las acusaciones eran mutuas y los testimonios contradictorios. En situaciones como éstas, la frontera entre la competencia y la actividad delictiva no estaba claramente establecida. Los derechos a las aguas eran complejos, así que la percepción y la reacción de los hechos variaban enormemente según la satisfacción de los usuarios. La presencia de la mafia convertía la competencia en algo más complicado. La entrada de un nuevo proveedor, según

4 ASPa, b. 3244, vol. 13 A, Verbale di dibattimento di prima istanza, 3/12/1929, p. 615, y Verbale di dibattimento di prima istanza, 31/10/1929, p. 201.

5 ASPa, b. 3240, vol. 1, Interrogatorio di Giovanni Fontana, 15/06/1926, p. 124.

6 ASPa, b. 3244, vol. 13 ${ }^{\text {A }}$, Verbale di dibattimento di prima istanza, 29/10/1929, pp. 151-155 y 21/11/1929, p. 438. 
los testimonios, tenía que obtener la aprobación de jefe de la organización criminal, que en ocasiones coincidía con el propietario o gestor de la máquina de elevación de la zona. ${ }^{7} \mathrm{La}$ ruptura del equilibrio hidráulico y económico, en el contexto social dominado por la mafia, podía desencadenar una guerra entre proveedores que perjudicaba el regadío y era percibida como una amenaza para los cultivos. ${ }^{8}$

Los distribuidores de las aguas sólo en raras ocasiones eran también los propietarios de las máquinas. Solían ser jornaleros o trabajadores en las tierras con pozos o empleados en pequeñas empresas privadas. Cuando acababa la temporada de riego volvían a otros oficios, generalmente vinculados con los trabajos agrícolas. Su sueldo era bajo e incluía el mantenimiento de los canales, pero solían especular con la actividad de riego. Jugaban con la cantidad de agua realmente suministrada, lo que afectaba sobre todo a las pequeñas propiedades. Pese a la habitual tolerancia de los agricultores ante estos pequeños robos de recursos, algunos testimonios denunciaban la presencia de guardias que, como Nicoló Gargano, uno de los mayores imputados, suministraban la mitad de lo acordado. ${ }^{9}$ Se trataba de algo excepcional que desencadenaba malentendidos o conflictos más graves.

Por su parte los guardas se defendían argumentando que no obtenían la remuneración pactada. Por ello, afirmaban, tenían que imponerse «enérgicamente» para recaudar el dinero de los suministros. ${ }^{10}$ Hay que tener en cuenta que, como guardias rurales, iban armados y provistos de licencia. ${ }^{11}$ No obstante, numerosos usuarios declararon que no hacía falta el uso de las armas: para mantener el control del territorio, de las cosechas y de las aguas, era suficiente la fama de violentos de los administradores del riego. Los proveedores podían operar un sistema arbitrario de precios y favorecer un agricultor frente a otros, ya que el miedo a represalia anulaba el riesgo de sufrir denuncias.

El agua para el regadío se configuraba así, no sólo como un medio para enriquecerse, sino también como una de las caras más evidente de la mafia siciliana (Lupo, 2004: 119). Ya en el último tercio del siglo XIX se hablaba de una «secta» de los administradores de las aguas, que impedía a los usuarios protestar ante la posibilidad de represalias y violencias. La capacidad de controlar el territorio y de crear redes sociales era funcional a la búsqueda y dominio de economías ilegales. Buena parte de la literatura sobre este tema afirma que esta dinámica de

7 La policía fascista describió, entre otros, los abusos de un jefe de la mafia de Villabate (entre Bagheria y Palermo). Ese administraba una máquina que durante décadas había fomentado la citricultura de las tierras cercanas. Aunque disponía de unas infraestructuras hidráulicas obsoletas, imponía a los usuarios — con la violencia directa e indirecta- aceptar sus suministros. ASPa, b. 3242, Sentenza Sezione d'accusa, p. 401; ASPa, b. 3244, vol. 13A, Verbale di dibattimento di prima istanza, 12/11/1929, p. 312.

8 ASPa, b. 3242, Sentenza Sezione d'accusa, p. 402

9 ASPa, b. 3244, vol. 13 A, Verbale di dibattimento di prima istanza, 6/11/1929, p. 256.

10 ASPa, b. 3242, Sentenza Sezione d'accusa, pp. 478-479, y b. 3244, vol. 13 ${ }^{\mathrm{A}}$, Verbale di dibattimento di prima istanza, $5 / 11 / 1929$, p. 223.

11 Algunas licencias de armas de los distribuidores están en guardados en ASPa, b. 3244, vol. 13C. 
especulación e intimidación perduró hasta décadas recientes (Damiani, 1884-1885: tomo I, fasc. II, 413; Caldo, 1984; Sciarrone, 1998).

Las organizaciones criminales querían insertar sus hombres de confianza en las posiciones claves del riego. Por ello, según las reconstrucciones policiacas, los empresarios que montaban una nueva máquina de extracción muchas veces renunciaban a la gestión directa por tener que favorecer a los colaboradores protegidos por la mafia. El negocio del regadío permitía alterar el mercado del trabajo y de la producción. Por ejemplo, en Ficarazzi (Figura 1), un pueblo en la ribera oeste del río Eleuterio, entre Palermo y Bagheria, un grupo de guardias provocó la ruina económica de un propietario: se le negó el riego de los limonares y sus árboles fueron dañados, perdió la cosecha y su patrimonio agrícola. El grupo que fue acusado de este abuso, los hermanos Bonetti, se encargaba de podar los árboles y de distribuir las aguas provenientes de un pequeño embalse en Scillato. ${ }^{12}$ El agua de ese acueducto, construido en 1880 , era más barata que la de los pozos. Sus concesionarios vendieron los sobrantes a los citricultores por lo menos hasta los años veinte, cuando el incremento demográfico obligó a que se destinaran exclusivamente hacia los centros urbanos (Arnao, 1899; Lo Meo, 2010). Santo Bonetti, encargado de administrar este recurso, pretendía obtener la gestión de las tierras y eliminar la competencia en la venta de aguas. ${ }^{13}$ Además, sus hermanos eran guardias de pozos cercanos, por lo que alcanzaron sin obstáculos el monopolio de las aguas en Ficarazzi. ${ }^{14}$

Las tensiones con los agricultores crecían en torno a la construcción, manutención y control de los canales de distribución. No todos respetaban los cambios en la red hidráulica que, además, suponían un coste elevado para los propietarios. De hecho, en la documentación consultada y en la literatura de toda la primera mitad del siglo veinte, se repiten las historias de abusos, de desvíos no autorizados y de destrucción de la red hidráulica. La apropiación de los recursos públicos y su venta arbitraria era algo corriente. Pero sólo ocasionalmente desencadenaba una reacción por parte de las víctimas por miedo a las represalias (Hess, 1973: 177-178).

En particular, las obras para mejorar la red hidráulica podían provocar reacciones violentas, especialmente entre los que no toleraban el paso por las tierras cercanas o pretendían limitar la competencia en el mercado del riego. Por ello, los canales podían sufrir un desvío, un daño o la completa destrucción, aunque a veces por razones legales. Los hermanos Bonetti, entre otras cosas, fueron acusados de haber pretendido un pago ilegal para construir algunos canales. ${ }^{15}$ En estos casos, pese a las denuncias de invasión de la propiedad y a las intimidaciones y extorsiones, tampoco la autoridad pública sabía cómo intervenir.

12 ASPa, b. 3240, vol. 1, Verbale della Polizia giudiziaria sull'associazione a delinquere, 24/07/1926, pp. 58-59 y Dichiarazione di Pietro Tempra, 26/06/1926, p. 112.

13 ASPa, b. 3245, Copia del Estratto di sentenza penale, [20/01/1930], p. 188.

14 ASPa, b. 3244, vol. 13 ${ }^{\mathrm{A}}$, Verbale di dibattimento di prima istanza, 25/11/1929, p. 478; b. 3240, vol. 1, Interrogatorio di Salvatore Macchiarella, 15/06/1926, p. 127.

15 ASPa, b. 3241, vol. 5, Verbale della P.G., 6/06/1927, pp. 220. 
Por su parte, los guardias del agua del río Eleuterio, en manos de una administración privada llamada casa S. Elia, admitieron haber pedido un pago extra para las operaciones de riego y la mejora hidráulica. ${ }^{16} \mathrm{~A}$ finales del siglo XIX, los usuarios pagaban un canon anual que les permitía obtener los suministros necesarios. No obstante, no era la administración central que pagaba el sueldo a los guardias, sino los usuarios que pagaban según las «horas» de agua recibida. ${ }^{17}$ En la primera posguerra, según la policía, las extorsiones en torno al riego aumentaron, especialmente cuando la administración del agua S. Elia instaló nuevos conductos o reparó los anteriores. Los guardias pretendieron que los agricultores cubrieran el coste de las reparaciones, ya que se habrían beneficiado de las obras de mejora. En cambio, los usuarios afirmaban que se trataba de un gasto a cargo de la administración S. Elia que recibía sus pagos anuales. ${ }^{18}$ En definitiva, sin otros recursos disponibles, todos tuvieron que pagar para asegurarse los suministros, pese a no ser propietarios de los canales, sino meros usuarios. Algunos testimonios hasta declararon que frente a las posibles represalias y a las amenazas armadas, cualquier intento de oposición al pago era inútil y peligroso.

¿Se trataba de un apoyo solidario a la comunidad o, como alegaba la policía fascista, de la demostración de la capacidad de intimidación de los guardias? Pese a la presencia de relaciones sociales densas y de larga duración, es difícil imaginar el desarrollo de una actitud cooperativa en ausencia de confianza, reputación y reciprocidad entre los actores de la gestión hidráulica (véase Fonseca, Montalba y García, 2015: 584). Las intenciones reales de usuarios y guardias en este caso nunca fueron aclaradas y el juicio acabó con la absolución de los guardias imputados. Situaciones como ésa demostraban la dificultad para solucionar los conflictos y los pleitos en ausencia de un marco jurídico claro y consensuado. Los usuarios se sentían víctimas de la arbitrariedad de la distribución hidráulica. Consideraban a algunos proveedores como los amos absolutos de las aguas, capaces de «cualquier delito». ${ }^{19}$

Esto nos impone una reflexión sobre algo que no se puede considerar como una serie de episodios sin conexión entre ellos, sino como la expresión de una desesperada o prepotente búsqueda de agua. Los agricultores, en muchas ocasiones, percibían las dinámicas de riego, sus turnos o su privación, como una injusticia. Sin embargo, ¿cómo se podía establecer quienes y en que medida tenían los derechos a las aguas de un proveedor particular? Teniendo en cuenta el supuesto uso de los canales como herramienta de prevaricación, ¿era posible obligar a un propietario de pozo a suministrar las aguas a otro agricultor?

La identificación entre guardas del agua u otros trabajadores del riego y organización criminal, como señalado por la policía fascista, podría ocultar que la misma gestión del riego

16 ASPa, b. 3245, Copia del Estratto di sentenza penale, [20/01/1930], pp. 126-127.

17 ASPa, b. 3241, vol. 4, Verbale della Polizia giudiziaria e allegati con testimonianze, 26/03/1927, p. 352; b. 3242, Sentenza Sezione d'accusa, pp. 480-481.

18 ASPa, b. 3240, App. al vol. 1, Memoriale di Clemente Alcamo, mayo 1929, p. 18; b. 3242, Sentenza Sezione d'accusa, pp. 349-350

19 ASPa, b. 3241, vol. 4, Verbale della Polizia giudiziaria e allegati con testimonianze, 26/03/1927, p. 354. 
era de por sí injusta y corrupta. El concepto de equidad en torno al uso de las aguas se moldea en base a las exigencias agrícolas particulares, a los conocimientos técnicos, a las ideas políticas e, incluso, a las redes familiares y a los contactos más o menos estrechos con los propietarios de las aguas (cf. Molle, 2008). La resolución de las controversias, sin la autoridad de un jurado de riegos o la documentación sobre los derechos al riego, se basaba en el miedo a las personas que imponían los turnos y los precios. La incertidumbre jurídica amparaba los actos criminales. Por ello, los policías distinguían con dificultad los procedimientos legales de venta de aguas de los delictivos, provocando la rabia y desesperación en los agricultores. ${ }^{20}$

Es determinante para entender las relaciones sociales en torno al riego que, según la defensa, la ausencia de un contacto directo con los usuarios exculparía automáticamente a los guardas del agua acusados de mafia. Los que supervisaban la distribución, que vigilaban las balsas o que trabajaban en el cuidado de los canales no podían abusar de su posición laboral; en cambio, la persona que se encargaba de distribuir las aguas directamente a los usuarios era prevenidamente considerado como mafioso. ${ }^{21}$

Ahora bien, no puede decirse que todos los distribuidores fueran miembros de la organización criminal. Los usuarios insatisfechos, que acusaban a los distribuidores de abusos o mafia, podían no tener en cuenta la escasez e irregularidad de los recursos. ${ }^{22}$ Pocos agricultores justificaban las actividades de sus proveedores como el resultado de una sabia regulación de las aguas. ${ }^{23}$ En cambio, algunos expertos en la materia, como algunos profesores agrónomos de Palermo interrogados durante el juicio, recordaban que para el riego de los limones en agosto no había suficiente agua, porque las solicitudes eran excesivas. Las disputas eran inevitables porque todos los usuarios querían obtener mayores caudales. ${ }^{24}$ Se estaba perpetrando, según estas interpretaciones, una demonización de los administradores del agua que organizaban los turnos teniendo en cuenta la geografía del territorio, los recursos disponibles y la capacidad de elevación de la máquina. ${ }^{25}$

En la documentación analizada, los tribunales no podían condenar a los intermediarios por no suministrar las aguas a los que no tenían unos derechos establecidos a esos recursos. Se trataba, pues, de favores o sencillamente de una transacción comercial, lo que justificaba la arbitrariedad de la gestión privada. El productor se encontraba a la merced de los propietarios

20 ASPa, b. 3240, vol. 1, Carta de Pietra Puleo al Procuratore, 24/08/1926, p. 194.

21 ASPa, b. 3244, vol. 13A, Verbale di dibattimento di prima istanza, 30/10/1929, p. 181; ASPa, b. 3245, Corte d'appello 1930, Motivi di ricorso di Lo Medico Benedetto, 29/11/1930, p. 539. ASPa, b. 3244, vol. 13C, Difesa di Lo Medico Benedetto, 9/01/1930, p. 331.

22 ASPa, b. 3240, vol. 1, Interrogatorio dell' imputato Girolamo Pagano, 1/08/1926, p. 765.

23 ASPa, b. 3244, vol. 13 , Verbale di dibattimento di prima istanza, 30/10/1929, p. 182.

24 ASPa, b. 3244, vol. 13' , Verbale di dibattimento di prima istanza, 5/12/1929, p. 653.

25 ASPa, b. 3244, vol. 13 ${ }^{\mathrm{A}}$, Verbale di dibattimento di prima istanza, 5/12/1929, p. 654 y 7/12/1929, pp. 707-708. 
de las máquinas de bombeo y de sus administradores, que tenía que respectar para asegurarse el riego de sus campos. ${ }^{26}$

En ausencia de organismos públicos o colectivos, emergió la desconfianza de los usuarios en el mercado y la gestión privada. No obstante, tampoco la institucionalización de la gestión hídrica evitó el riesgo de especulaciones y conflicto. La policía judicial afirmaba que los abusos estaban vinculados a la gestión del agua, tanto directa, como a través de una administración compleja, y tanto de las subterráneas, como las de superficiales. ${ }^{27}$

\section{LOS CONSORCIOS DE PROPIETARIOS: ¿ESPERANZAS FRUSTRADAS?}

Especialmente a partir de la primera posguerra, el Estado italiano intervino para solucionar los problemas relacionados con el riego, como el desorden hidráulico, el mercado de las aguas y los abusos en la distribución. Junto con este «proyecto de bonifica», se impulsó la formación de consorcios de propietarios para la mejora rural y la ordenación de los canales y de su uso colectivo (Stampacchia, 1983: 29). En los años veinte y treinta del siglo XX, estos organismos - tradicionales en la Italia del Norte - se difundieron también en Sicilia, especialmente en las zonas dedicadas a los cultivos intensivos. En este caso, la casa S. Elia, que ya hemos mencionado, se convirtió en una administración colectiva de las aguas del río Eleuterio.

Se modificaba una gestión hídrica que remontaba al siglo XVI y que, ya desde finales del XIX, involucraba todas las tierras citrícolas cercanas en propiedad o arriendo. Los agricultores, principalmente de Bagheria y Ficarazzi, adquirían el derecho a caudales fijos tras el pago de una cuota anual, que variaba según la extensión de la propiedad y la antigüedad del convenio. ${ }^{28}$ Sin embargo, la administración de la casa S. Elia, que se encargaba de la manutención de los canales y de la distribución, se volvió paulatinamente confusa y corrupta. Por esta razón, los propietarios pretendían evitar esa intermediación y, en 1921, crearon el Consorcio Eleutero o S. Elia (Lo Meo, 2010: 70). El paso a la gestión colectiva fue lastrado por la injerencia de los antiguos propietarios. Se inició antes de la Primera Guerra Mundial y no concluyó hasta 1944, tras un largo recurso judicial que apartó definitivamente a los antiguos concesionarios (Schifani, 1969: 35).

La propia administración colectiva fue controvertida desde sus inicios. El primer consejo de administración se disolvió por dimisiones y el prefecto de Palermo asumió el mando. La injerencia estatal mermó la eficacia del Consorcio y su capacidad de representar a los agricultores. No obstante, hay que señalar que durante el juicio de 1929 algunos imputados alegaron su esfuerzo para la creación del consorcio como evidencia de su lucha «para qui-

26 ASPa, b. 3242, Sezione d'accusa, Processi alligati n. 260, pp. 478-479.

27 ASPa, b. 3240, vol. 1, Verbale della Polizia giudiziaria sull'associazione a delinquere, 24/07/1926, pp. 61-62.

28 ASPa, b. 3242, Sentenza Sezione d'accusa, p. 113 
tarla legalmente de las manos de la mafia». ${ }^{29}$ Incluso la policía pensaba que la promoción y consolidación de un consorcio de riego se podía considerar como una herramienta eficaz para luchar contra el dominio criminal de los recursos, por lo que sus fundadores quedaban, en cierta medida, automáticamente absueltos.

La nueva administración presumió de una gestión más ordenada y no permitió el pago de los guardias por parte de los usuarios. ${ }^{30}$ Sin embargo, los guardias que trabajaban para la casa S. Elia continuaron sus actividades bajo la administración del Consorcio Eleutero. Esta continuidad se confirmó también con las denuncias de extorsión que apagaron el entusiasmo de los agricultores. ${ }^{31}$ La falta de normas consensuadas y de turnos capaces de satisfacer a los usuarios hizo que estos se dirigieran hacia nuevos proveedores, tanto de aguas subterráneas, como de los embalses de reciente construcción. El Consorcio abandonó la manutención de los canales y a partir de 1955 se quedó sin aguas ni usuarios. (Schifani, 1969: 34-44). La gestión colectiva no había cumplido con las expectativas de sus fundadores y se desveló el sustrato social que perpetuaba el control abusivo de las aguas.

Entre los diversos intentos locales de crear nuevas instituciones, que pretendían revolucionar la gestión del riego, destacó el caso del Consorcio Idro-Agricolo de Bagheria (CIAB). Las críticas principales a las acciones de este organismo fueron tres: en primer lugar, su escasa eficacia en la trasformación del secano; en segundo lugar, la continuidad entre los administradores de las máquinas privadas y los empleados por el consorcio; en tercer lugar, la falta de unas normas consensuadas sobre las elecciones internas.

El CIAB nació en 1922 tras una reunión de más de 500 agricultores, en su mayoría productores de limones y vecinos de Bagheria. Liderados por algunos agrónomos cooperativistas, los agricultores expresaron «desde abajo» su voluntad de acabar con el monopolio privado de las aguas y con la injerencia mafiosa. Pretendían crear un organismo que fuera capaz de mantener unos precios justos y sostenibles para los productores. ${ }^{32}$ Este se encargaría de mejorar la red hidráulica del territorio, ejecutando unas importantes obras de canalización. Asimismo, gestionaría las aguas de un embalse hidroeléctrico que se estaba construyendo en las colinas del interior, cerca de Piana degli Albanesi, y que se inauguró en $1923 .{ }^{33}$

Los agricultores de Bagheria se empeñaron en una relación monopolista con la empresa constructora, la Società Anonima Siciliana per l'Irrigazione (SASI). No sólo se compro-

29 ASPa, b. 3240, Ap. al vol. 1, Difesa dei Macchiarella, s.f.; b. 3244, vol. 13 A, Verbale di dibattimento di prima istanza, 3/12/1929, p. 610; b. 3245, Copia del Estratto di sentenza penale, [20/01/1930], pp. 255-256.

30 ASPa, b. 3240, vol. 1, Interrogatorio di Giovanni Fontana, 15/06/1926, p. 124.

31 ASPa, b. 3242, Sentenza Sezione d'accusa, p. 497; ASPa, b. 3241, vol. 4, Verbale della Polizia giudiziaria e allegati con testimonianze, 26/03/1927, p. 359.

32 Archivo privado del Consorzio Idro-Agricolo de Bagheria (ACIAB), c. Atti costitutivi e decreto, Atto costitutivo rilasciato dall'archivio notarile e presentato alla commissione straordinaria, 1924 (copia de 1964).

33 Sobre la realización de este embalse y la intervención de la mafia, véase los estudios de Giuseppe Barone (1986, pág. 175 y ss.) y de Manoela Patti (2008: 80). 
metieron a pagar un mínimo anuo, sino que el uso de cualquier otra fuente hídrica tenía que obtener la autorización de la SASI. ${ }^{34}$ Se trataba de una intermediación controvertida, pero prometía la llegada de caudales regulares $-\mathrm{y}$ a precios más asequibles y progresivos- $\mathrm{y}$ una alternativa al sistema privado de búsqueda y distribución de las aguas. Los fundadores pretendían revolucionar, conscientemente, los equilibrios socio-económicos locales en un perímetro comunitario de $22 \mathrm{~km}$ y un total de 600 ha que incluían los términos municipales de otros pueblos (se excluyeron las abastecidas por el consorcio S. Elia). ${ }^{35}$ Esta definición de las fronteras fue una actividad incompleta y continua - y hasta cruel— que, indirectamente, aspiraba a la construcción una identidad comunitaria (cfr. Bauman, 2003: 17).

Efectivamente, las aguas del embalse y el nuevo consorcio de riego revolucionaron los equilibrios sociales e hidráulicos del territorio. Los fundadores y las autoridades públicas procuraban que los agricultores se consideraran miembros de la entidad, no sólo meros clientes. Desde el principio, el prefecto de Palermo patrocinó las actividades del CIAB. Para empezar, en 1925 firmó un decreto para obligar a todos los propietarios de las tierras incluidas dentro del perímetro del consorcio a pagar las cuotas anuales y una contribución inicial que provocó numerosas protestas. ${ }^{36}$ Cada usuario pagaba por un suministro mínimo independientemente de su disfrute, que el CIAB cobraba para alcanzar el importe pactado con la SASI. ${ }^{37} \mathrm{El}$ prefecto pretendía evitar que sólo una parte de los usuarios pagaran, es decir, eliminar la presencia de free-riders que, como teorizó Garret Hardin (1968), harían fracasar la gestión colectiva de los bienes comunes.

Por un lado, el reconocimiento de la obligatoriedad dio fuerza y amparo legal al nuevo organismo; por otro, provocó el malhumor de los agricultores involucrados..$^{38}$ Entre ellos destacaban los proveedores de las aguas subterráneas, que no querían perder a sus clientes. Las obras de canalización se revelaron especialmente conflictivas, ya que los propietarios de las aguas las percibieron como una competencia desleal que nada tenía a que ver con la real transformación del secano a regadío. ${ }^{39}$ Las protestas se multiplicaron rápidamente, sobre todo entre aquellos agricultores que tenían ya otros recursos para regar sus fundos o que, por razones productivas, preferían mantener mayor flexibilidad. Tras una reunión de usuarios, en 1928, hasta se solicitó la abrogación del decreto que impuso la obligatoriedad del consorcio

34 ACIAB, c. Contratto x la fornitura d'acqua tra la SASI e il Consorzio 1926 e 1957, Contratto del '26, art.13.

35 ACIAB, c. Altre deliberazioni, Compenso per la compilazione del catasto, 30/06/1926.

36 ACIAB, c. Espropri risarcimento danni, Pagamento paraguanto 7/05/1926.

37 ACIAB, c. Sentenza utenza 12/11/1929.

38 ACIAB, c. Sentenza utenza, Sentenza sull'utenza, 12/11/1929.

39 ACIAB, c. De Cordova, Atto consortile 02/08/1926; Proprietari danneggiati dal canale 23/09/1926; c. Rosalia Parisi di Torrebrun, Lettera al prefetto, 22/12/1930. 
y la drástica reducción de los costes de riego. ${ }^{40}$ Finalmente, sólo los que pudieron demostrar ser usuarios de las aguas S. Elia o que disponían de recursos subterráneos para regar lograron quedar al margen del consorcio. ${ }^{41}$

En definitiva, centenares de agricultores no aceptaron la injerencia de una entidad que, no sólo consideraban inútil en un contexto de relaciones sociales y económicas ya consolidado, sino que no reconocían ni como propia, ni como un sistema de gestión hídrica eficaz y duradero. ${ }^{42}$ Aun así, el precio bajo del agua del embalse, la cobertura hidráulica casi total y la obligatoriedad garantizaron un cierto éxito a la nueva administración. ${ }^{43}$ El CIAB protagonizó las relaciones sociales y económicas en torno a la gestión hídrica durante décadas. Sin embargo, la entidad se caracterizó, especialmente en los años veinte y treinta, por una cierta inestabilidad administrativa y por el recelo de los agricultores hacia la regulación del riego (D'Amaro, 2010).

Los propietarios solicitaban el suministro a la administración central, que decidía el orden de las reservas y lo comunicaba al jefe de los distribuidores (Schifani, 1969: 44). Los encargados de regular los suministros a los usuarios eran guardias jurados armados, empleados del Consorcio, que no tuvieron un reglamento hasta 1953.44 Los agricultores no tenían que pactar los suministros con ellos, ni pagarlos directamente. Sin embargo, su figura era controvertida. Por un lado, se reconocía su centralidad en el desarrollo de la producción citrícola; por otro, se percibía la falta de control que permitía sobornos, robos y abusos. En línea con lo que hemos dicho en el apartado anterior, la relación entre agricultores y los encargados de riego mantuvo su carácter conflictivo, pero el miedo a las represalias garantizaba el silencio de los usuarios del organismo que se declaraba comunitario y democrático (D'Amaro, 2012). Asimismo, los usuarios acusaban sus administradores de perpetrar el dominio, aunque sólo indirecto, de las organizaciones mafiosas en las huertas. Efectivamente, la administración fascista envío a menudo comisarios extraordinarios para sustituir al presidente y al CdA, acusados de corrupción u otros crímenes. ${ }^{45}$

Asimismo, los usuarios vivieron los procedimientos electorales como un procedimiento inútil y una imposición desde arriba. Los estatutos, aprobados sólo en 1928, fueron adaptados a las normas nacionales de los consorcios. No se trataba, pues, de normas consensuadas entre

40 ACIAB, c. Decreti Prefettura, Relazione dell'adunanza degli agricoltori contro il consorzio per il prezzo troppo alto dell'acqua e la successiva risposta critica del Regio commissario del Cia 1928; c. Reclami utenza, Reclami utenza contro acqua assegnata, 1929

41 ACIAB, c. Reclami ammortamento.

42 ACIAB, c. Centro di coordinamento consorzi, Usufruizioni dell'acqua Torrebruna, Reclamo Puleo Carmelo 26/01/1928.

43 ACIAB, c. Altre deliberazioni, Completamento della rete idrica consorziale 4/04/1933.

44 ACIAB, c. Altre deliberazioni, Nomina di sorvegliante, 15/06/1926; c. regolamento organico, Regolamento del Corpo delle Guardie giurate del Consorzio idro-agricolo, 1954.

45 ACIAB, c. Elezioni, Pratica ricostituzione amministrazione ordinaria 29-3-1936 XIV, Lettera del Prefetto al commissario prefettizio, 26/10/1935. 
los usuarios, sino impuestas por los dirigentes que, en realidad, habían acelerado la creación de la entidad para acceder a las ayudas estatales destinadas a las obras de canalización (Barone, 1986; cfr. Ostrom, 1990: 93). En particular, la elección del CdA, que tenía las verdaderas funciones directivas, concedía más poder a los miembros con mayores extensiones de tierra. No sólo porque sus votos tenían más peso, sino porque obtenían la delegación de los otros miembros a través de un sistema clientelar. ${ }^{46}$ Pese al nuevo contexto democrático y a las luchas campesinas de las décadas centrales del siglo XX —-dinamizadas especialmente por el Partido Comunista - el sistema electoral nunca fue modificado sustancialmente ${ }^{47}$ (véase un ejemplo en la Tabla 1). Los pequeños agricultores acabaron percibiendo el CIAB como una «administración parasitaria»: ${ }^{48}$ una entidad que no les representaba, en el que no tenían voz y que imponía turnos ilógicos y tasas indeseadas.

Tabla 1. Ejemplo de asignación de voto en el CIAB, según los estatutos de 1965 (Fuente: $\mathrm{ACIAB}$ )

\begin{tabular}{|c|c|c|c|c|c|c|}
\hline $\begin{array}{c}\text { Horas de agua } \\
\text { asignada a cada } \\
\text { empresa }\end{array}$ & $\begin{array}{l}\text { Núm. de } \\
\text { empresas }\end{array}$ & $\begin{array}{c}\text { Horas de } \\
\text { aguas totales } \\
\text { en cada } \\
\text { grupo }\end{array}$ & $\begin{array}{c}\text { Número de } \\
\text { votos para } \\
\text { cada empresa }\end{array}$ & $\begin{array}{l}\text { Número total } \\
\text { de votos en } \\
\text { cada grupo }\end{array}$ & $\begin{array}{c}\text { Superficie } \\
\text { total en } \\
\text { «tumolo» } \\
\text { 140ha. }\end{array}$ & $\begin{array}{l}\text { Superficie en } \\
\text { hectáreas }\end{array}$ \\
\hline de 1 a 10 & 1775 & 10.705 & 1 & 1.775 & 1.338 & 186.575 \\
\hline de 11 a 20 & 752 & 10.932 & 2 & 1.504 & 1.366 & 190.479 \\
\hline de 21 a 34 & 343 & 9.069 & 3 & 1.029 & 1.134 & 158.128 \\
\hline de 35 a 50 & 133 & 5.532 & 4 & 532 & 692 & 96.495 \\
\hline más de 50 & 128 & 19.494 & $\begin{array}{l}\text { 1voto por } \\
\text { cada } 8 \text { horas } \\
\text { de agua }\end{array}$ & 2.148 & 2.436 & 339.683 \\
\hline total: & 3131 & 55.732 & & 6.988 & 6.966 & 971.360 \\
\hline
\end{tabular}

Finalmente, hay que subrayar la importancia de las aguas subterráneas en el desarrollo agrícola del territorio analizado. A partir de la Segunda Posguerra, la mejora tecnológica, que potenció la capacidad de captación de las bombas, estaba incrementando el peso de las aguas subterráneas en diversos territorios del Mediterráneo (Calatayud y Martínez, 2005).

46 ACIAB, c. Statuti vecchi 1926, Copia dello Statuto inviato al Commissario Prefettizio, 31/01/1926.

47 ACIAB, Assemblea straordinaria, 15/10/1967.

48 ACIAB, Verbale dell'assemblea generale ordinaria, 29/05/1966. 
En 1953, considerando exclusivamente la zona de regadío, que constituía el 39,2 \% de la superficie agraria de Bagheria, sólo el 18,6 \% (971,36 ha), estaba incluido en el perímetro del consorcio, es decir, aprovechaba las aguas del embalse. Otra pequeña parte, 13,4 \% (358 ha) disfrutaba de otros recursos superficiales, de los pequeños ríos cercanos. Finalmente, el $68 \%$ (1.843 ha) del regadío sobrevivía gracias a los 21 pozos del territorio (Schifani, 1955: 44).

Por otra parte, hay que señalar que la citricultura dependía de las máquinas administradas por agentes privados, no sólo en competencia con el CIAB, sino también como principales proveedores de sus miembros. A partir de los años cuarenta, los administradores del consorcio tuvieron que buscar la colaboración de los propietarios de las máquinas para hacer frente al incremento de la demanda de agua. Los que se habían configurado como los principales opositores de la gestión colectiva, vendieron sus recursos al consorcio que los redistribuía garantizando a los agricultores consorciados unos precios más estables y unitarios. ${ }^{49}$ Resulta evidente que hay que redimensionar los efectos «revolucionarios» del consorcio que, no sólo no logró la plena confianza de sus miembros, sino que nunca los liberó completamente del mercado del agua.

\section{Conclusiones}

La historia que hemos contado en la primera parte de este trabajo puede contribuir a arrojar luz sobre un matiz no muy explorado de la gestión hídrica en las comunidades locales. El control mafioso del riego es un ejemplo histórico de como un recurso natural, un bien común, puede configurarse como una herramienta de uso privado, no sólo de enriquecimiento, sino también de violencia y de poder social. Es complicado interpretar las controversias en torno a las aguas por la misma peculiaridad de su gestión y uso, y por los límites ambientales. Los administradores de las máquinas justificaban con la escasez hídrica la distribución, que los usuarios percibían como arbitraria y prepotente. ${ }^{50}$ Por lo tanto, lo que para los agricultores era un abuso del guardia del agua para esos era un capricho del usuario. La frontera entre relaciones sociales conflictivas y verdaderos episodios de injerencia criminal quedaba borrosa y controvertida.

En la huerta de Palermo se había enraizado un control industrial y privado de los recursos hídricos. El mercado del agua excluía del derecho al riego a los agricultores más pobres. Los citricultores no eran, pues, ni usuarios de una administración pública, ni miembros de una comunidad de gestión colectiva. Eran más bien clientes, que compraban las aguas en un régimen de semimonopolio en el que no hacía falta establecer vínculos con los otros agricultores, que eran competidores en el abastecimiento hídrico. No estaban acostumbrados a

49 ACIAB, c. Altre deliberazioni, Prezzo acqua irrigua, 14/11/ 1942.

50 ASPa, b. 3244, vol. 13 ${ }^{\mathrm{A}}$, Verbale di dibattimento di prima istanza, 31/10/1929, p. 190. 
tener responsabilidad sobre el mantenimiento de las máquinas o sobre la sostenibilidad del uso de los recursos.

El Estado, especialmente a partir de los años veinte, incentivó la gestión comunitaria, basada en unas normas consensuadas y más eficaces de gestión del riego. Efectivamente, la asamblea del CIAB se planteaba como una herramienta de gestión democrática: todos los miembros podían participar en ella. Sin embargo, el diseño institucional es sólo una de las variables que influyen sobre la gestión de los comunes: como sugiere Agrawal (2003), en el análisis hay que incorporar el papel de la política, las dinámicas del poder, los factores ambientales, etc. La instrumentalización política de la entidad, el incremento de la importancia proporcional de las aguas subterránea, a partir de los años cuarenta y la escasa identificación de los usuarios con el CdA deterioró la confianza de los agricultores en su administración.

Entre otras cosas, para la aceptación plena el consorcio faltó la creación de un nuevo tipo de capital social, es decir, una relación de confianza y reciprocidad entre los usuarios y entre estos y los administradores. Son elementos intangibles, pero «esenciales para que se completen muchas transacciones complejas en la vida moderna» (Ostrom y Ahn, 2003: 182; cf. Uphoff, 2005) y, en particular, para la cooperación y el funcionamiento de las instituciones de riego —especialmente si las aguas escasean. Para los agricultores de Palermo, el agua tenía un coste elevado que había que tener en cuenta para la producción. No era considerado un bien común, que había que redistribuir según las exigencias, sino un recurso económico, una mercancía que había que acapararse antes que los demás para el éxito de la cosecha. La llegada de los consorcios, pues, no supuso una fractura, sino un solapamiento de propiedades hidráulicas y de derechos de aguas. Por ello, el agua era todavía percibida como instrumento de control político y social de la comunidad local, tanto si la vendía un administrador de las máquinas de extracción, como si la proporcionaba un consorcio de riego amparado por el Estado.

\section{REFERENCIAS}

Agrawal, A. (2001): Common Property Institutions and Sustainable Governance of Resources. World Development, 29(10), 1649-1672.

Agrawal, A. (2003): Sustainable Governance of Common-Pool Resources: Context, Methods, and Politics. Annual Review of Anthropology, 32, 243-262.

Alfonso, F. (1875): Sui sistemi di d'irrigazione nell'agro palermitano. En Atti della società di acclimazione e di agricoltura in Sicilia, pp. 17-35.

Alfonso, F. (1877): Trattato di idraulica agraria. Tip. P. Montaina \& Comp., Palermo.

Arnao, E. (1899): La coltivazione degli agrumi. Alberto Reber, Palermo.

Barone, G. (1986): Mezzogiorno e modernizzazione. Elettricità, irrigazione e bonifica nell' Italia contemporanea. Einaudi, Torino. 
Barone, G. (1987): Egemonie urbane e potere locale (1882-1913). En Aymard, M. y Giarrizzo, G. (eds.): Storia d'Italia. Le regioni dell' unità a oggi. La Sicilia. Einaudi, Torino, pp. 189-370.

Bauman, Z. (2003): Comunidad: en busca de seguridad en un mundo hostil. Siglo XXI de España, Madrid. Bernardi, E. (2014): L'agricoltura, i tecnici e la bonifica integrale. Studi Storici, 55(1), 81-92.

Bevilaceua, P. (1989): Le rivoluzioni dell'acqua. Irrigazione e trasformazioni dell'agricoltura tra Sette e Novecento. En Bevilacqua, P. (ed.): Storia dell'agricoltura italiana in età contemporanea (Vol. I. Spazi e paesaggi). Marsilio, Venezia, pp. 255-318.

Bevilacqua, P. (1996): Tra natura e storia. Ambiente, economie, risorse in Italia. Donzelli, Roma.

Blando, A. (2008): L'avvocato del diavolo. Meridiana, 63, 53-78.

Boelens, R. y Vos, J. (2014): Legal pluralism, hydraulic property creation and sustainability: the materialized nature of water rights in user-managed systems. Current Opinion in Environmental Sustainability, 11, 55-62.

Calatayud Giner, S. (1990): Los inicios de la mecanización en el regadío valenciano, 1850-1930. Areas, 12, 203-211.

Calatayud Giner, S. y Martínez Carrión, J. (2005): El cambio tecnológico en el uso de las aguas subterráneas en la España del siglo XX. Un enfoque regional. Revista de Historia Industrial, 28, 81-116.

CAldo, C. (1973): Le «giarre» delle pianure costiere palermitane. En Atti del XXI congresso geografico italiano, Verbania, 13-18 settembre 1971 (Vol. III). Novara, pp. 71-81.

Caldo, C. (1984): Mafie e tecniche irrigue in Sicilia. En Caldo, C. (ed.): La città globale. Palumbo, Palermo, pp. 143-155.

Cavallo, F. L. (2011): Terre, acque, macchine. Geografie della bonifica in Italia tra Ottocento e Novecento. Diabasis, Reggio Emilia.

Chironi, G., Crescimanno, M., Fardella, G., Schifani, C. y Tudisca, S. (eds.) (1987): L'agrumicoltura mediterranea e le prospettive italiane. Settore Economia del Dipartimento di Economia, Ingegneria e Tecnologie Agrarie dell'Università degli Studi di Palermo, Palermo.

Cusimano, G. (1989): Dinamica degli schemi colturali e tipologie areali nella provincia di Palermo. Uomo e territorio, 4, 87-151.

Cusimano, G. (1990): Le geometrie dell'acqua: territorio e agrumi nel bagherese. En Cusimano, G. (ed.): Geografia e cultura materiale. Flaccovio, Palermo, pp. 89-119.

D'Amaro, F. (2010): Il controllo delle acque irrigue. La fondazione del Consorzio idroagricolo di Bagheria (anni venti del XX sec.). I frutti di Demetra, 22, 5-19.

D’Amaro, F. (2012): Il mercato dell'acqua. Politica, istituzioni e conflitti nel distretto agrumicolo di Bagheria (XX sec.). Meridiana, 71-72, 271-291.

Damiani, D. (1884-1885): Inchiesta Damiani. En Jacini, S. (coord.): Atti della giunta per la inchiesta agraria e sulle condizioni della classe agricola (Vol. XIII). Roma.

Drago, V. (1972): Inchiesta in un paese dove fioriscono i limoni: acqua, mafia e lotta tra le classi a Bagheria, Inchiesta, II, 8, 7-21.

Ferri Ramírez, M. y SANChis Ibor, C. (2001): De comuners a regants: comunitat, territori i conflicte a les hortes valencianes. Afers, 40, 641-654. 
Fonseca, F., Montalba, R. y García, M. (2015): Redes sociales, capital social y acción colectiva en dos territorios campesinos de la región de La Araucanía, en Chile, para enfrentar problemas asociados al acceso al agua. Papers, 100(4), 577-606.

Glick, T. F. (1970): Irrigation and Society in Medieval Valencia. The Belknap Press of Harvard University, Cambridge (Mass.).

González Alcantud, J. (1998): Economía moral del agua. Demófilo: Revista de cultura tradicional, 27, 198-208.

Grando, S. y Volpi, G. (2014): Backwardness, modernization, propaganda. Agrarian policies and rural representations in the Italian Fascist regime. En L. Fernández-Prieto, P.-M. JuAn Luis y C. Miguel: Agriculture in the Age of Fascism. Authoritarian Technocracy and Rural Modernization, 19221945. Brepols, Turnhout (Bélgica), pp. 43-83.

Hardin, G. (1968): The tragedy of the commons. Science, 162, 1243-1248.

Hermosilla Pla, J. (Ed.). (2010): Los regadíos históricos españoles: paisajes culturales, paisajes sostenibles. Ministerio de Medio Ambiente y Medio Rural y Marino, Madrid.

Hess, H. (1973): Mafia. Laterza, Bari.

Lanaro, S. (1989): Da contadini a Italiani. En Bevilaceua, P. (coord.): Storia dell'agricoltura (Vol. III. Mercati e istituzioni). Marsilio, Venezia, pp. 935-968.

Lo Meo, V. (2010): Il limone perduto. I centocinquant'anni della limonicoltura nel comprensorio di Bagheria. Il Nuovo Paese, Bagheria.

Lupo, S. (1987): Tra società locale e commercio a lunga distanza: la vicenda degli agrumi siciliani. Meridiana, 1, 81-112.

Lupo, S. (1990): Il giardino degli aranci. Il mondo degli agrumi nella storia del Mezzogiorno. Marsilio, Venezia.

Lupo, S. (2004): Storia della mafia dalle origini ai giorni nostri. Donzelli, Roma.

Lupo, S. (2008): Introduzione. Mafia e fascismo. Meridiana, 63, 9-14.

Lupo, S. (2018): La mafia. Centosessant'anni di storia. Donzelli, Roma.

MaAss, A. y Anderson, R.L. (1978): ... and the desert shall rejoice: conflict, growth and justice in arid environments. The MIT Press, Cambridge.

Molle, F. (2008): Nirvana concepts, narratives and policy models: Insights from the water sector. Water Alternatives, 1(1), 131-156.

Molle, F. y Closas, A. (2017): Groundwater Governance: A Synthesis. Groundwater Governance in the Arab World. International Water Management Institution, WMI project publication, Colombo Sri Lanka.

Olson, M. (1965): The logic of Collective Action. Public Goods and Theory of Group. Harward University Press, Cambridge-Massachusetts.

Ostrom, E. (1990): Governing the commons. The evolution of institutions for collective action. Cambridge University Press, Cambridge.

Ostrom, E. у Анn, T.K. (2003): Una perspectiva del capital social desde las ciencias sociales: capital social y acción colectiva. Revista Mexicana de Sociología, 65(1), 155-233. 
Pantaleone, M. (1971): Il sasso in bocca. Mafia e Cosa Nostra. Cappelli, Bologna.

Pardo Nacher, C. (2014): Conqueridors del secà: el procés de fundació de les primeres societats civils de reg a la Plana de Castelló (1897-1914). Millars, XXXVII, 121-148.

Patтi, M. (2008): Sottoprocesso. Le cosche palermitane. Meridiana, 63, 73-94.

Pezzino, P. (1987): Stato violenza società. Nascita e sviluppo del paradigma mafioso. En Aymard, M. y Giarrizzo, G. (coords.): Storia d'Italia. Le regioni dell'unità a oggi. La Sicilia. Einaudi, Torino, pp. 903-982.

Rochefort, R. (1961): Le travail en Sicilie. Étude de géographie sociale. Presses Universitaire de France, Paris.

Ruf, T. (2010): Dynamismes comparés et contrastes des sociétés paysannes méditerranéennes et de leurs territoires hydrauliques. En Wolfer, B. A. (ed.): Agricultures et paysanneries du monde. Mondes en mouvement, politiques en transition. Quae, Versailles, pp. 145-184.

Sanchis-Ibor, C., Boelens, R. y García-Mollá, M. (2017): Collective irrigation reloaded. Re-collection and re-moralization of water management after privatization in Spain. Geoforum, 87, 38-47.

Sciarrone, R. (1998): Il capitale sociale della mafia. Relazioni esterne e controllo del territorio. Quaderni di sociologia, 18, 51-72.

Schifani, C. (1969): Aspetti economici dell'irrigazione in provincia di Palermo. En C. Schifani, Chironi, G. y Bacarella, A. (eds.): Utilizzazione e costi dell'acqua per irrigazione in Sicilia. Istituto di economia e politica agraria dell'Università di Palermo, Palermo, pp. 13-78.

Scrofani, S. (1962): Sicilia: utilizzazione del suolo nella storia, nei redditi e nelle prospettive. ESA, Palermo.

Stampacchia, M. (1983): Tecnocracia e ruralismo. Alle origini della bonifica fascista (1918-1928). ETS Pisa, Pisa.

Stampacchia, M. (2000): «Ruralizzare l'Italia!». Agricoltura e bonifiche tra Mussolini e Serpieri (19281943). Franco Angeli, Milano.

Uphoff, N.T. (2005): Social Capital and Irrigation Management: Bringing Rigor and Evidence to the Relationship. En Sнivaкоті, G. P. (ed.): Asian Irrigation in Transition. Responding to Challenges. Sage Publications, Nueva Delhi, pp. 79-98.

VERri, C. (2008): Un dibattito marxista: mafia e latifondo. Meridiana, 63, 135-156.

Wittfogel, K. (1966): Despotismo oriental. Estudio comparativo del poder totalitario. Guadarrama, Madrid.

Cómo citar este artículo:

D’Amaro, F. (2018). Pozos y mafiosos. La inacabada transición del mercado del agua a la gestión colectiva en el Palermo del siglo XX. Cuadernos de Geografía, 101, 71-90.

https://doi.org/10.7203/CGUV.101.13721

\section{(C) $(1) \Theta \Theta$}

Este obra está bajo una licencia de Creative Commons Reconocimiento-NoComercial-SinObraDerivada 4.0 Internacional. 